\title{
Recurrent Laryngeal Nerve Injury
}

National Cancer Institute

\section{Source}

National Cancer Institute. Recurrent Laryngeal Nerve Injury. NCI Thesaurus. Code C103171.

Damage to the recurrent laryngeal nerve. 